\title{
Tabularia
}

TABULARIA Sources écrites des mondes normands médiévaux Guillaume de Volpiano : Fécamp et l'histoire normande | 2002

\section{Les femmes dans l'histoire du duché de Normandie}

Women in the history of ducal Normandy

\section{Elisabeth Van Houts}

\section{OpenEdition}

Journals

Édition électronique

URL : http://journals.openedition.org/tabularia/1736

DOI : 10.4000/tabularia.1736

ISSN : 1630-7364

Éditeur :

CRAHAM - Centre Michel de Boüard, Presses universitaires de Caen

Référence électronique

Elisabeth Van Houts, «Les femmes dans I'histoire du duché de Normandie », Tabularia [En ligne], Guillaume de Volpiano : Fécamp et l'histoire normande, mis en ligne le 10 juillet 2002, consulté le 01 mai 2019. URL : http://journals.openedition.org/tabularia/1736; DOI : 10.4000/tabularia.1736 


\title{
Les femmes dans l'histoire du duché de Normandie Women in the history of ducal Normandy
}

\author{
Elisabeth VAN HOUTS \\ Emmanuel College, Cambridge \\ CB2 3AP, UK \\ emcv2@cam.ac.uk
}

Résumé:

Loin de conclure que les femmes normandes n'ont joué aucun rôle dans le processus de l'écriture de l'Histoire, je voudrais suggérer qu'elles ont pu s'impliquer de différentes manières dans l'histoire de leurs familles. Elles ont agi comme des " canaux de communication historique » entre les générations. Des femmes de l'aristocratie, s'occupant du passé, du présent et du futur, ont stimulé la production de récits historiques et prophétiques. Trois dossiers en particulier ont retenu mon attention : le don du château du Homme (Cotentin) de la comtesse Adeliza de Bourgogne aux religieuses de La Trinité de Caen en 1075; les deux versions du récit de la fondation de l'église de Saint-Martin d'Auchy de la fin du $\mathrm{XI}^{e}$ siècle et le manuscrit Paris BnF lat. 5390 (Raoul Glaber, Vie de Guillaume de Volpiano; Adson de Montier-en-Der, L'origine et du temps d'Antéchrist et la prophétie sibylline) copié entre 1060 et 1070 .

Mots-clés: généalogie, prophétie, historiographie médiévale, femmes, douaire, Aumale, Raoul Glaber, Adson de Montier-en-Der, Guillaume de Volpiano, Mathilde de Normandie.

\begin{abstract}
:
Instead of concluding that Norman women did not play any role in the process of writing history, I should like to suggest that there were other ways in which they could be involved with the history of their families. They acted as channels of historical knowledge between the generations. Aristocratic women, preoccupied with the past, the present and the future, stimulated the production of historical and prophetical narratives. Three dossiers in particular will be discussed here: the grant of the castle of Le Homme (Cotentin) by Countess Adeliza of Burgundy to the nuns of Holy Trinity at Caen in 1075, the two versions of the foundation narrative of the church of St Martin at Auchy dating to the late eleventh century, and manuscript Paris BN Lat. 5390 (Ralph Glaber, Life of William of Volpiano; Adso of Montier-en-Der, The Origin and Time of the Antichrist and the sibyline prophecy) copied between 1060 and 1070.
\end{abstract}

Keywords: genealogy, prophecy, medieval historiography, women, dower land, Aumale, Ralph Glaber, Adso of Montier-en-Der, William of Volpiano, Matilda of Normandy. 
Au Moyen Âge, connaître l'Histoire et la transmettre était avant tout la tâche des clercs. Aux XI et XII ${ }^{\mathrm{e}}$ siècles, les historiens normands les plus fameux furent Dudon de Saint-Quentin, Guillaume de Jumièges, Guillaume de Poitiers, Orderic Vital et Robert de Torigni. Tous étaient des hommes ${ }^{1}$. Nous ne connaissons malheureusement pas d'historiennes normandes du Moyen Âge, quoique l'Histoire nous fournisse de nombreux exemples de femmes qui s'intéressèrent à l'histoire du duché. Dudon de Saint-Quentin suggère par exemple, que dans la production de son histoire des premiers ducs normands, la comtesse Gunnor $(\dagger 1031)$ joua un rôle ${ }^{2}$; Robert de Torigni laisse entendre que l'impératrice Mathilde († 1167), fille du duc/roi Henri (1100-1135), lui avait demandé de faire un ajout biographique aux Gesta Normannorum Ducum de Guillaume de Jumièges ${ }^{3}$. À son tour, Mathilde aurait pu être inspirée, dans cette commande, par l'exemple de sa mère la reine Mathilde II ( $† 1118)$, qui demanda de l'information sur ses ancêtres à l'historien anglais Guillaume de Malmesbury ${ }^{4}$, et qui avait reçu une biographie anonyme de sainte Marguerite d'Ecosse $(† 1095)$, sa mère, la grand-mère de l'impératrice Mathilde ${ }^{5}$. Ainsi la comtesse Gunnor, la reine Marguerite et les deux reines Mathilde témoignent de manière très convaincante qu'il existait un public de femmes nobles pour l'historiographie médiévale ${ }^{6}$.

Les sœurs monastiques de ces femmes, les moniales, s'intéressaient aussi à l'Histoire. Elles possédaient dans leurs bibliothèques des livres historiques, des généalogies et des chroniques sur l'histoire de leurs établissements ${ }^{7}$. Parmi les religieuses de Normandie, on trouve une femme écrivain, l'abbesse Marsilia de SaintAmand à Rouen, qui composa une histoire du miracle opéré, sur une femme de son monastère, par saint Amand, patron du lieu ${ }^{8}$. Ainsi, loin de conclure que les femmes normandes n'ont joué aucun rôle dans le processus d'écriture de l'Histoire, je voudrais suggérer qu'il y a d'autres manières où elles purent s'impliquer dans l'histoire de leurs familles d'origine et de celles où elles entraient. Si elles ne

1. SHOPKOW, 1997. Pour l'histoire du genre, voir VAN HOUTS, 1999 et VAN HouTs, 2000, p. 83-94.

2. Dudon De SAINT-QUENTIN, De moribus et actibus primorum Normanniae ducum, éd. Jules LAIR, Caen, Mémoires de la Société des Antiquaires de Normandie, t. XXIII, 1865, p. 289.

3. The Gesta Normannorum Ducum of William of Jumièges, Orderic Vital and Robert of Torigni, éd. Elisabeth VAN HouTs, Oxford, Clarendon Press (Oxford Medieval Texts), 1992, I, p. LXXXIIILXXXIV [ci-après GND].

4. Guillaume De Malmesbury, Gesta Regum Anglorum, éd. Roger Aubrey Baskerville MynORS, Rodney Thomson, Michael Winterbottom, Oxford, Clarendon Press (Oxford Medieval Texts), 1998-1999, t. 1, p. 2-5.

5. HUNEYCUTT, 1990 , p. 81-98.

6. JOHNSON, 1991, p. 144-147 sur l'éducation et les bibliothèques en France médiévale; il manque une étude sur les nonnes et l'historiographie en Normandie. Pour l'Allemagne ottonienne, voir CORBET, 1986.

7. VAN HOUTS, 1992 , p. 53-68.

8. VAN HouTs, Elisabeth, "L'oralité dans l'hagiographie normande au $\mathrm{XI}^{\mathrm{e}}$ et $\mathrm{XII}^{\mathrm{e}}$ siècles », in Les Saints dans la Normandie médiévale, Actes du colloque de Cerisy-la-Salle (26-29 septembre 1996), éd. Bouet Pierre, Neveux Francois, Caen, Presses Universitaires de Caen, 2000, p. 83-94; PlaTELLE, 1967, p. 83-106. 
jouaient pas un rôle d'écrivain, elles agissaient comme des canaux de communication historique entre les générations, pouvaient mémoriser des événements historiques et renseigner des historiens. Nous verrons aussi que leur implication dans l'Histoire et dans l'écriture de celle-ci était intimement liée au désir de contrôler leur présent et de connaître leur futur. En résumé, des femmes de l'aristocratie, s'occupant du passé, du présent et du futur, ont stimulé la production de récits historiques et prophétiques.

\section{Le passé}

Je commencerai par le passé, en examinant deux documents normands du $\mathrm{XI}^{\mathrm{e}}$ siècle qui prouvent que des femmes de l'aristocratie furent parfois les uniques sources d'informations historiques concernant leurs familles. Le premier document est une charte de la comtesse Adeliza, fille du duc normand Richard II (992$1026)$ et de Judith $(\dagger 1018)$, veuve du comte Renaud de Bourgogne $(\dagger 1056)^{9}$. En 1075 , la vieille comtesse bourguignonne - elle avait alors plus de soixante-dix ans - fit don aux religieuses de la Trinité de Caen de son château du Homme (aujourd'hui l'Isle-Marie) dans le Cotentin. La charte fournit de précieux détails sur les événements qui eurent lieu quarante ans auparavant et qui ne se trouvent pas dans les chroniques ducales de Guillaume de Jumièges ou de Guillaume de Poitiers. Adeliza dit explicitement qu'elle acheta le château avec son or (de auro suo) à son frère le duc Robert le Magnifique (1027-1035), donc avant la mort de celui-ci, quatre décennies auparavant. À mon avis, l'achat du château par la comtesse bourguignonne remonte probablement à la fin du règne de Robert, du temps où il rassembla des fonds pour financer son pèlerinage à Jérusalem ${ }^{10}$. L'acquisition du château serait aussi liée à la présence en Normandie de son fils Gui, un cousin contemporain du fils du duc Robert, Guillaume le Conquérant, qui fut élevé à la cour normande ${ }^{11}$. Si le château du Homme fut conçu comme un pied-à-terre pour Gui, il est très intéressant de voir que dans le document caennais, sa mère Adeliza affirme explicitement qu'elle le perdit à la suite de l'appropriation injuste par son fils Gui ${ }^{12}$. Nous avons ici, sans doute, une référence explicite à la révolte de Gui contre son cousin Guillaume pendant les années 1047-1050. Selon la version de l'histoire d'Adeliza, Gui, à son tour, aurait donné le château à son ami et collaborateur Néel II de Saint-Sauveur ${ }^{13}$. Celui-ci revendiquait son droit sur le

9. BATES, David, Regesta Regum Anglo-Normannorum. The Acta of William I (1066-1087), Oxford, Clarendon Press, 1998, n 58, p. 270. [Ci-après Regesta...]

10. GND, II, p. 80-81 ; sur le financement du pèlerinage, voir MUSSET, 1962, p. 149-150.

11. GND, II, p. 120-123; The Gesta Guillelmi of William of Poitiers, éd. Ralph Henri Carless DAVIS, Marjorie CHIBNAll, Oxford, Clarendon Press (Oxford Medieval Texts), 1998, p. 8-13, p. 32-35, p. 42-43.

12. BATES, Regesta..., n 58, p. 270 : Quod postea Guido, filius suus iniuste sibi auferens, dedit illud Nigello vicecomiti.

13. Voir note $\mathrm{n}^{\circ} 12$. 
château qu'il avait gardé jusqu'à présent, c'est-à-dire jusqu'en 1075. De son côté, Adeliza contrait l'argument de Néel en soulignant que le père de Néel (Néel Ier) avait conservé le château en tant qu'officier ducal, à titre de vicomte, et non pas comme sa possession héréditaire ${ }^{14}$. La présence de la comtesse/reine Mathilde I ${ }^{\text {ère, }}$ femme de Guillaume le Conquérant, à l'occasion du don du château à la Trinité de Caen, est la preuve que le duc soutenait la version de l'histoire du château racontée par sa tante la comtesse Adeliza, et non pas celle présentée par son vicomte, Néel II de Saint-Sauveur ${ }^{15}$.

Mais pourquoi ce don du château au monastère de la Trinité en 1075? Il me semble que la comtesse Adeliza essaya de contrôler le destin du château du Homme avant sa mort, sans doute avec le consentement de son cousin le duc Guillaume. Par ce don aux moniales de Caen, comme Pierre Bauduin l'a signalé, elle retirait d'une circulation future parmi les femmes de la famille ducale un domaine anciennement donné en douaire ${ }^{16}$. Le domaine du Homme avait probablement fait partie d'un douaire depuis la mère d'Adeliza, Judith, car Le Homme se trouvait sans doute parmi des domaines du Cotentin donnés par Richard II à sa femme, et après par Richard III à sa fiancée Adèle de France ${ }^{17}$. Or, Adèle avait épousé en secondes noces Baudoin V de Flandre et paraît avoir emporté son droit sur Le Homme avec elle ${ }^{18}$. Toutefois, entre 1026 et 1075, l'histoire du domaine du Homme n'est signalée que par la charte d'Adeliza de Bourgogne. Dans ce contexte, il est très intéressant de noter qu'ensuite notre information sur cette localité se trouve dans une autre charte du monastère de la Trinité, datée de 1082, qui déclare que Le Homme ne reviendra pas immédiatement aux religieuses, mais passera d'abord à une autre femme de la famille ducale, en l'occurrence la comtesse Adélaïde d'Aumale (voir en annexe le tableau généalogique, p. 34), fille de Robert le Magnifique, sœur de Guillaume le Conquérant et donc nièce de la comtesse Adeliza de Bourgogne, qui se vit garantir à titre viager la possession du $\operatorname{burgus}^{19}$. Se pose alors la question: pourquoi la comtesse Adeliza de Bourgogne essaya-t-elle de sortir le château du

14. BATES, Regesta..., n 58, p. 270 : Si autem prefatus Nigellus dixerit se hoc iure hereditario habuisse, sciendum est quia pater eius hoc aliter minime habuit, nisi quia vicecomes erat eiusdem patrie, et precepit sibi comitissa Adeliz ut sibi inde veluti minister serviret ; DELISLE, 1867, p. 4-6.

15. Pour la présence de Mathilde, voir BATES, Regesta..., p. 92-93.

16. Je remercie Pierre BAUDUIN de m'avoir fourni une copie de son article avant publication.

17. Fauroux, Marie, Recueil des actes des ducs de Normandie de 911 à 1066I, Caen, Mémoires de la Société des Antiquaires de Normandie, t. XXXVI, 1961 [ci-après Recueil...], n 11, p. 82-85 (Brix, p. 85 «Bruet») et $\mathrm{n}^{\circ} 58$, p. 180-182 (p. 182 : castella que ibi habentur, videlicet Carusburg cum eo quod dicitur Holmus et eo quod dicitur Brusco cum iis que ad hec aspicere videntur) ; BAUDUIN, à paraître, [p. 8, 12].

18. BAUDUIN, à paraître [p. 13].

19. BATES, Regesta..., n 59, p. 271-86 à la p. 278: donavimus burgum de Hulmo cum reditibus suis Adelissa amita mea [sc. Rex Willelmus I] benigniter annuente, cuius hereditas erat, sed et comitissa A (delissa) de Albemarla concedente, eo videlicet pacto ipsa teneret in vita sua, post obitum vero suum ad victum sanctimonialium sancta possideret ecclesia. On peut noter, avec P. Bauduin, que Le Homme est cité parmi les biens usurpés à la Trinité de Caen sous le règne de Robert Courteheuse (WALMSLEY, Charters, p. 127 : et Hulmum aufertur Sancte Trinitati iniuste). 
Homme de la circulation des biens dévolus aux femmes de la famille ducale à l'occasion des transactions matrimoniales?

Peut-être la réponse a-t-elle été fournie par l'un des témoins du don de 1075, Baudoin, fils du comte Gislebert, un autre membre de la famille ducale de Normandie. Aux environs de 1050, il épousa une femme issue de la famille ducale que nous n'avons pas identifiée, mais qui est décrite par Orderic Vital comme une fille de la tante (paternelle) du duc Guillaume le Conquérant ${ }^{20}$. Elle serait soit une fille d'Adeliza de Bourgogne, soit une fille de la sœur d'Adeliza (que les sources normandes ne nomment pas) qui épousa Baudoin IV de Flandre ${ }^{21}$. Il est donc important pour nous de réaliser que la femme inconnue de Baudoin, fils du comte Gislebert, était une petite-fille du duc Richard II, qui aurait eu quelque droit sur le château du Homme, soit parce qu'elle l'avait reçu en douaire, soit parce qu'elle était parente directe de Robert le Magnifique. Après la mort prématurée de cette épouse, Baudoin, fils du comte Gislebert, se maria en secondes noces avec une femme nommée Emma (après 1086). Il est possible que Baudoin ait été présent, à l'occasion du don de la comtesse Adeliza, à cause des droits des enfants que Baudoin avait eus de sa première femme, la nièce d'Adeliza, et parce que son consentement au transfert du château aux religieuses de la Trinité était nécessaire. Quoi qu'il en soit, Le Homme n'arriva jamais aux mains des religieuses parce qu'entre 1075 et 1082, la comtesse Adélaïde d'Aumale exerça son droit sur le château, probablement comme fille du duc Robert le Magnifique: plus tard en effet, au XII ${ }^{\mathrm{e}}$ siècle, Le Homme figure comme le douaire de sa petite fille, une autre Adélaïde d'Aumale. Par conséquent, même si les femmes de la famille ducale normande ne pouvaient pas toujours contrôler le destin de ces douaires tant qu'elles étaient en vie, elles pouvaient faire rédiger des documents pour leur transmission, dans lesquels elles fournissaient des détails historiques qui expliquaient l'acquisition, l'histoire et le destin des domaines dont elles avaient été dotées. L'information féminine, qui est souvent de type privé, est pour nous une source parallèle à l'information publique fournie par l'historiographie ducale.

Je voudrais à présent porter mon attention sur la comtesse Adélaïde d'Aumale, un autre exemple de femme dans ce rôle d'informatrice sur la connaissance du passé. La comtesse Adélaïde, qui était la sœur du duc Guillaume par leur père le duc Robert le Magnifique, naquit avant $1035^{22}$. Elle est célèbre pour ses

20. The Ecclesiastical History of Orderic Vitalis, éd. Marjorie CHIBNALL, Oxford, Clarendon Press (Oxford Medieval Texts), 1969-1978, IV, p. 208 [ci-après ORDERIC VITAL, H. E.] : et filiam amitae suae uxorem dedit [sc Guillemus dux]. Elle fut la première femme de Baudoin, nommée Albreda dans un document de v. 1340, voir Dugdale, V, p. 377.

21. Fauroux, Recueil... n 59, 61 ; MUSSET, 1967, n² 2, 8, 11 ; DOMESDAY BOOK, I, f. 107 : uxor Balduini.

22. Il n'y a aucun doute qu'Adélaïde fut une fille de Robert le Magnifique et donc une demi-sœur de Guillaume le Conquérant, voir, ORDERIC VITAL, $H$. E. II, 264 : filiam scilicet Rodberti ducis. Elle a été identifiée comme sœur utérine (soror uterina) - dans le sens de demi-sœur paternelle - par Robert de Torigni (GND, II, p. 272). Cf. aussi Robert de Torigni sur Guillaume d'Eu, frère utérin de Richard II par leur père, Richard I (GND, II, p. 128). Dans sa Chronique, Robert de Torigni (Chronicle..., éd. Richard HowletT, p. 24) identifie Adélaïde comme unam filiam nomine Aeliz de alia concubina. 
trois mariages: le premier en 1053 avec le comte Enguerrand II de Ponthieu $(† 1054)$, qui lui donna une fille, également nommée Adélaïde (II ${ }^{23}$, le second avec le comte Lambert de Lens dont est issue une autre fille nommée Judith, connue surtout par son mariage avec l'earl anglais Waltheof $(\dagger 1076)^{24}$; le troisième et dernier, entre 1065 et 1070, avec le comte Eudes de Champagne (après 1118), le père de son fils Etienne ${ }^{25}$. Elle avait donc trois enfants de trois époux: deux filles et un fils. Adélaïde Ière a été appelée Adélaïde d'Aumale en souvenir du comté d'Aumale au nord-est du duché de Normandie, qui constituait peut-être un douaire venu de son premier mari Enguerrand II de Ponthieu ${ }^{26}$. Elle le transféra à ses autres époux, une coutume rare, mais pas unique dans l'histoire médiévale ${ }^{27}$. L'influence d'Adélaïde I⿳亠े⿵冂丶 forte à en juger par le dossier des documents concernant l'église collégiale de Saint-Martin d'Auchy à Aumale, qui nous fournissent de précieux détails sur le rôle d'Adélaïde I ${ }^{\text {èr }}$ dans la transmission de l'histoire familiale de son premier mari et sur celle du comté d'Aumale.

Le dossier est constitué de deux documents, qui représentent deux versions racontant l'histoire de la fondation de l'église de Saint-Martin d'Auchy, nommés A et $\mathrm{B}^{28}$ : la version A présente le droit exercé par Etienne, fils d' Adélaïde İ̀re, sur le comté d'Aumale ${ }^{29}$ et la version B celui de la fille aînée d'Adélaïde, Adélaïde II $^{30}$. Les deux versions s'accordent sur le fait que l'église de Saint-Martin a été fondée par le «vir nobilis Guerenfridus », l'homme noble Guerenfroid, au temps du duc Richard II - donc entre 996 et 1026 - et elles énumèrent des dons d'Adélaïde I ${ }^{\text {ère, }}$ de Judith, la sœur d'Adélaïde, et d'autres seigneurs locaux. La version d'Etienne (A) souligne que celui-ci confirme les dons de ses parents, sa mère, la très noble comtesse Adélaïde, sœur du duc Guillaume, et son mari le comte Eudes ${ }^{31}$. Sans date, mais remontant aux années 1090, la version A n'indique que vaguement le lien d'héritage entre Guerenfroid, Adélaïde İ̀re et son troisième mari, le père d'Etienne. Cependant la version B, c'est-à-dire la version d'Adélaïde II, est plus détaillée que la version $\mathrm{A}$ en ce qui concerne le lien précis entre les générations et surtout entre l'homme noble Guerenfroid et Adélaïde I ${ }^{\text {ère }}$. La charte B affirme que

23. GND, II, p. 270-271.

24. Vita Waldevi, éd. D. GILES, p. 10 : Juettam, filiam comitis Lamberti de Lens. Cette source est le seul témoin pour l'identification du second époux d'Adélaïde.

25. GND, II, p. 270 ; ORDERIC VITAL, H. E., II, 264-4 ; IV, 182-183.

26. Pour l'histoire d'Aumale, voir BAUDUIN, 1998, p. 404-431. Je remercie Pierre Bauduin de m'avoir fourni une copie de sa thèse en microfiche. Pour une histoire anglaise, voir ENGLISH, 1979, p. 1417.

27. LE JAN, 1995, p. 369 ; p. 371 et BAUDUIN, à paraître [p. 13-15].

28. Je suis Pierre Bauduin pour les sigles utilisés pour ces documents.

29. MUSSET, 1961, p. 32-35; BAUdUIN, 1998, p. 405.

30. StAPleton, 1836, p. 358-60 ; BAUdUin, 1998, p. 406.

31. MussET, 1961, p. 32 : jussu Addelidis nobillissime comitisse sororis sicilicet Willelmi regis anglorum que ideo apicibus commendari voluit, confirmante viro suo, videlicet Odone comite, una con filio suo Stephano... 
le premier mari d'Adélaïde, le comte Enguerrand II de Ponthieu, est le fils d'une femme nommée Berthe, et que cette Berthe est la fille de Guerenfroid ${ }^{32}$. En détaillant les liens de parenté entre Enguerrand et son grand-père Guerenfroid, la fille d'Enguerrand, Adélaïde II, mettait l'accent sur son ascendance directe, par son père Enguerrand, avec sa grand-mère paternelle Berthe, l'héritière de Guerenfroid. En outre, le document $\mathrm{B}$ donne à Adélaïde II le même titre que sa mère: $c o$ mitissa, et se réfère donc à elle comme comtesse Adélaïde, fille de la comtesse Adélaïde I ̀̀re, qui après la mort de ses parents succéda à « l'imperium» d'Aumale ${ }^{33}$. Il est évident que la charte remonte à une période où Adélaïde II avait été reconnue comme l'héritière d'Aumale, une position semblable à celle de sa grand-mère paternelle Berthe, fille de Guerenfroid.

Comment pouvons-nous concilier les deux versions du dossier d'Aumale qui représentent les droits contradictoires des deux enfants d'Adélaïde I ${ }^{\text {ère }}$ ? La question est fascinante parce que nous savons qu'Etienne survécut à sa sœur aînée et qu'il gouverna Aumale jusqu'à son exil en dehors de Normandie en $1127^{34}$. Dans quelle mesure et à quelle période Adélaïde II eut-elle besoin de souligner sa posi-

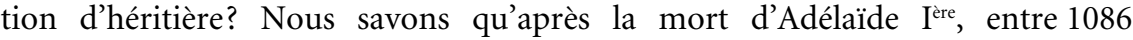
et $1090^{35}$, Etienne, peut-être avec son père Eudes, fut chargé de la défense d'Aumale en $1089 / 1090^{36}$. Nous savons aussi qu'il donna une charte à l'abbaye Saint-Lucien de Beauvais en juillet 1096 avec le consentement de sa sœur Adélaïde II $^{37}$ et qu'il gouverna Aumale après son retour de Jérusalem en 1100 jusqu'à son exil. Je voudrais suggérer qu'Adélaïde II profita de l'absence de son demi-frère Etienne pendant la première croisade quand il accompagna le duc Robert Courteheuse (1087-1106, 1134) à Jérusalem entre septembre 1096 et $1100^{38}$. Pendant cette période, Adélaïde II eut la chance de gouverner le comté et de revendiquer son droit comme enfant unique d'Enguerrand de Ponthieu, seigneur d'Aumale. Sans mari et sans enfants, elle n'avait aucune chance de prolonger la dynastie de sa mère, et elle n'aurait remplacé Etienne que temporairement comme régente. Pendant sa

32. STAPLETON, 1836, p. 358 : jussu Enguerrani consulis qui filius Bertae supradicte Guerenfridi filie et Delidis comitisse uxoris sue, sororis scilicet Willelmi regis...

33. STAPLETON, 1836, p. 358 : Et quicquid beneficii eidem ecclesie supradicti antecessores concensserunt, Addelidis comitissa supradicti Engueranni et supradicte Adelidis filia, que post obitum illorum in inperio successit, confirmat.

34. ENGLISH, 1976, p. 15.

35. Adélaïde I⿳亠丷厂re était encore en vie en 1086 (FARLEY (éd.), Domesday Book II, fol. 91b [Essex] and 431b [Suffolk]), mais morte en 1089/1090 quand son fils Etienne (ORDERIC VITAL, H. E., IV, p. 182) ou son mari Eudes (The Chronicle of John of Worcester, éd. Reginald Ralph DARLINGTON, Patrick MCGURCK, Jennifer BRAY, Oxford, Clarendon Press (Oxford Medieval Texts), 1995-1998 [ci-après JEAN DE WORCESTER], III, p. 57) sont dits être en possession d'Aumale. Elle est de manière certaine décédée en 1096 quand sa mort est mentionnée dans une charte de son fils pour Saint-Lucien de Beauvais (voir ci-dessous, note $\mathrm{n}^{\circ} 37$ ).

36. Voir note $\mathrm{n}^{\circ} 35$.

37. GALLIA Christiana, XI, Instr. XV, col.19-20 : consensu simul et corroboratione sororis meae Adelidis.

38. DAVID, 1920, p. 228 ; ENGLISH, 1976, p. 15. 
régence, Adélaïde II eut l'opportunité de réviser le document de la fondation de Saint-Martin d'Auchy pour donner des détails sur son origine, en tant qu'héritière d'Enguerrand II. L'information sur la généalogie de ses ancêtres, fournie sans doute par sa mère Adélaïde I, témoigne de l'importance qu'attachait Adélaïde II à son droit d'héritière sur Aumale et de l'importance de la connaissance de l'histoire pour prouver ce droit.

Nous ne savons pas quand Etienne revint de Jérusalem ni à quel moment précis il reprit le gouvernement d'Aumale. Les sources provenant des domaines anglais de l'héritage d'Adélaïde Ire suggèrent que mon hypothèse d'une régence d'Adélaïde II n'est pas valide. En 1095-96, le comte Eudes et son fils Etienne furent impliqués dans une révolte contre le roi Guillaume le Roux; quelques Anglais proposèrent à cette occasion le jeune Etienne comme roi (sans doute en qualité de petit-fils de Robert le Magnifique) ; l'affaire provoqua l'incarcération du comte Eudes et le départ d'Etienne pour la croisade ${ }^{39}$. À cause de cette révolte, le roi donna les domaines anglais, le comté d'Holderness en particulier, à Arnulf de Montgommery, qui le gouverna jusqu'à sa propre révolte en $1102^{40}$. Il existe donc une possibilité réelle pour que pendant six ans, de 1096 à 1102, le comté d'Aumale ait été aux mains d'Adélaïde II, pas en tant que régente à la place d'Etienne, mais à titre personnel. Les circonstances de la restitution d'Aumale à Etienne ne sont pas connues ${ }^{41}$.

Ironiquement, après la mort des deux Adélaïde d'Aumale, il émergea une troisième Adélaïde, la petite fille d'Adélaïde I, fille d'Etienne et de sa femme Hawise de Mortemer. Cette Adélaïde reçut un douaire dont nous avons déjà parlé, en l'occurrence Le Homme en Cotentin. Elle ne donna pas le château, mais l'église du Homme à l'abbaye de Saint-Sauveur-le-Vicomte, la fondation de la famille de Néel de Saint-Sauveur, l'ancien adversaire de la comtesse Adeliza de Bourgogne ${ }^{42}$. Le témoignage du douaire d'Adélaïde III explique donc que si Adélaïde I fit honorer le don de Le Homme aux religieuses de Caen par sa tante Adeliza de Bourgogne, elle ne l'a fait que pour le château et pas pour l'église. Indirectement elle avait contribué à rediriger la possession d'une partie du domaine du Homme de la famille ducale et comtale vers l'abbaye familiale de la famille de Saint-Sauveur, ennemie de longue date de sa tante.

Les deux exemples du château du Homme et du comté d'Aumale montrent que la connaissance historique n'était pas seulement importante pour les hommes, mais aussi, et peut-être surtout, pour les femmes, parce que la connaissance de la généalogie familiale et de la transmission des domaines familiaux était essentielle pour connaitre les droits des membres masculins et féminins de la famille, et

39. JEAN DE WORCESTER, III, p. 76-77.

40. ENGLish, 1976, p. 13-14 et FARrer, 1916, III, p. 26-32, avec une généalogie familiale à la p. 87.

41. DAVID, 1920, p. 158 signale qu'en 1104, Etienne, en tant que comte d'Aumale, salua le roi Henri $\mathrm{I}^{\mathrm{er}}$ pendant sa visite en Normandie (ORDERIC VITAL, H. E., VI, p. 56).

42. BAUDUIN à paraître [p. 12, n. 76] et ROUND, 1899, nº 971, p. 346-347. 
surtout, essentielle pour que les femmes exercent leurs droits en l'absence des hommes. Mes deux exemples soulignent aussi le fait que s'intéresser au passé n'était pas exclusivement du domaine masculin. Au contraire, l'implication féminine dans l'histoire et la généalogie familiale constituait une tâche qui demandait la collaboration des hommes et des femmes au Moyen Âge.

\section{Le futur}

Je terminerai sur l'engagement féminin vis-à-vis du futur. Par rapport au passé, l'engagement féminin était directement lié à cette implication dans le présent et le futur. L'angoisse du futur s'inspirait des récits prophétiques qui sont en réalité des images en miroir des récits historiques. Retournons à la Bourgogne du temps de la comtesse Adeliza, de son mari le comte Renaud de Bourgogne et aussi du temps de l'abbé Guillaume de Volpiano ( $† 1031$ ), dont le colloque de Fécamp commémore la venue. Son contemporain l'abbé Adson de Montier-en-Der ${ }^{43}$ est l'auteur de l'un des plus fameux textes prophétiques du Moyen Âge, De ortu et tempore Antechristi ${ }^{44}$. Composé à la demande de la reine Gerberge de France, l'arrière-grand-mère du comte Renaud, le traité sur l'Antéchrist est une petite histoire de l'ennemi de Dieu, et aussi une tentative d'établir la date de la fin du monde. On peut expliquer la requête royale et l'intérêt de la reine Gerberge pour le problème de l'Apocalypse en considérant l'angoisse de la reine pour l'avenir des enfants qu'elles avait eus de deux unions différentes. Les enfants de son premier mariage avec le comte Gislebert de Lorraine (†939) étaient menacés par le roi Louis IV, le père de ses autres enfants ${ }^{45}$. Pour guider sa stratégie maternelle, la reine Gerberge avait besoin d'information sur le futur. Grâce à sa protection, l'abbé Adson lui fournit ce texte. Dans le contexte de notre colloque sur Guillaume de Volpiano il est très intéressant de constater que le manuscrit le plus ancien de texte sur l'Antéchrist est aussi le manuscrit le plus ancien de la Vie de l'abbé Guillaume composée par Raoul Glaber ${ }^{46}$ et enfin pour un troisième texte qui les accompagne, une prophétie sibylline ${ }^{47}$. L'importance de ce manuscrit (Paris, BnF, ms lat. 5390) est d'autant plus grande si nous réalisons qu'il est une copie exécutée entre 1060 et 1070 à Fécamp par ordre de l'abbé Jean de Ravenne (102878) ${ }^{48}$. Sans doute l'autorité de Guillaume de Volpiano comme celle d'Adson de

43. Il n'est pas certain que les deux hommes, Guillaume et Adson, se soient rencontrés. Je remercie Neithard Bulst pour l'information donnée sur ce point.

44. Adso Dervensis de ortu et tempore antichristi necnon et tractatus qui ab eo dependunt, éd. Daniel VERHELST, Corpus Christianorum continuatio Mediaevalis, XLV, Turnhout, Brepols, 1996, p. 20-21.

45. LE JAN, 1995, p. 369-370.

46. Rodulfus Glaber Opera, éd. John France, Neithard Bulst, Paul ReYNOLDS, Oxford, Clarendon Press (Oxford Medieval Texts), 1989, p. 254-299, [ci-après Rodulfus].

47. SACKUR, 1898, p. 177-187.

48. Ibidem, p. XCIV-XCV et BULST, 1974, p. 453-454. 
Montier-en-Der et de l'abbé Jean de Ravenne, qui connaissait bien Guillaume de Volpiano, soulignent la valeur des textes prophétiques. La réputation spirituelle des trois abbés aurait donc donné une valeur supplémentaire aux textes prophétiques composés et copiés pour des reines et des femmes de l'aristocratie.

En considérant la date de la copie du manuscrit de Fécamp, entre 1060 et 1070, je voudrais suggérer, comme je l'ai fait il y a deux ans, au colloque sur la Tapisserie de Bayeux, que les trois textes auraient été copiés pour la duchesse Mathilde, femme du Conquérant, au temps de la conquête normande de l'Angleterre ${ }^{49}$. La duchesse Mathilde, comme la reine Gerberge, avait beaucoup de raisons de se faire du souci pour son mari, qui préparait son invasion de l'Angleterre, pour ses enfants présents et surtout à venir. Elle était particulièrement angoissée du fait qu'elle était enceinte de sa fille Adèle, née au commencement de l'année suivante (1067), même si la naissance à venir était vue comme un présage favorable. Les femmes de l'aristocratie et surtout les mères étaient toujours intéressées par le futur, appréciant beaucoup les textes prophétiques et recherchant des conseils des prophètes. Par exemple, selon Orderic Vital, la même duchesse Mathilde consulta un prophète allemand pendant les périodes prolongées de désaccord entre son mari Guillaume et son fils aîné Robert Courteheuse pour connaître le futur de la dynastie normande ${ }^{50}$.

Les craintes de Mathilde pour son mari et pour son fils émanaient de son impuissance à contrôler le présent et le futur. Dieu avait charge du futur mais on avait besoin des prophètes pour expliquer les signes de Dieu. La peur et l'angoisse $\mathrm{du}$ futur se cristallisent sur les moments de changements dynastiques. L'année 1066 fut l'un d'eux et il y en eut d'autres à la fin du XI' siècle et au début du XII siècle quand les fils du Conquérant, Robert Courteheuse, Guillaume le Roux et Henri Ier, connurent des désaccords. Le temps des fils du Conquérant fut marqué par une crainte pour l'avenir avec notamment le danger réel d'un changement dynastique. L'auteur de la Brevis Relatio, une petite histoire sur Guillaume le Conquérant, composée entre 1114 et 1120, avait inclus la prophétie suivante : le lignage ducal, qui s'était étendu sur sept générations, de Rollon à Henri Ier (11061135), devait subir une période de mésaventures pendant la génération suivante ${ }^{51}$. Ces indications avaient aussi de l'intérêt pour les hommes de la cour d'Henri Ier pour la connaissance du futur, surtout après la mort du fils de ce dernier, Guillaume Adelin, en 1120. L'absence d'héritiers nés de son épouse brabançonne, Adeliza de Louvain, constituait un sujet d'angoisse profonde ${ }^{52}$. Mais les témoins les plus anciens des horoscopes se réferaient à la génération suivante, pendant le

49. VAN HOUTS, à paraître; je voudrais mentionner le fait que Neithard BULST n'est pas d'accord avec ma suggestion parce que les trois textes mentionnés ont été réunis dans un livre manuscrit intitulé In Omeliis Gregorii, c'est un ensemble purement monastique et donc, selon Neithard BULST, pas opportun pour une reine.

50. ORDERIC VITAL, H. E., III, p. 104-108.

51. VAN HOUTS, 1997, p. 42-44.

52. WERTHEIMER, 1995, p. 100-115; STAFFORD, 2000, p. 4-27. 
règne du roi Etienne en Angleterre et le principat du duc Geoffroy d'Anjou en Normandie ${ }^{53}$. L'idée des sept générations heureuses est aussi présente dans les Gesta Normannorum Ducum, l'histoire des ducs normands, de Rollon à Guillaume le Conquérant, qui est en effet divisée en sept livres, un livre pour chacun des ducs. La division posa un problème monumental à Robert de Torigni, le rédacteur des Gesta, à qui l'impératrice Mathilde demanda d'ajouter un livre sur son père Henri Ier. Pour éviter d'écrire un livre sur la huitième génération ducale et d'encourager ainsi la prophétie d'une future catastrophe pour ces ducs, Robert porta le nombre de livres de huit à sept, en considérant le premier livre comme une introduction non numérotée. Par conséquent, le second livre devenait le premier livre et le livre supplémentaire, sur Henri Ier, le septième ${ }^{54}$.

La peur d'une catastrophe dynastique après la septième génération n'était pas une frayeur exclusivement normande, parce qu'une semblable angoisse est constatée parmi des membres de la famille capétienne au XII ${ }^{\mathrm{e}}$ siècle. Il est très intéressant que cette peur soit déjà présente, bien que sous forme implicite, dans la prophétie sibylline recopiée dans le manuscrit de Fécamp. Ce texte contient de longs passages sur les lignages dynastiques d'empereurs, de rois et de princes anonymes. L'anonymat des lignages transformait le texte en un support idéal pour des interprétations spécifiques à chaque type de public. Toute femme et tout homme qui avait une copie de la prophétie sibylline pouvait donc identifier de vagues événements mentionnés dans le texte à des faits de son propre temps. Par conséquent, cette femme ou cet homme pouvaient croire qu'ils connaissaient le futur et agir pour le mieux sur lui. Depuis les temps classiques, les Sibylles avaient toujours été associées aux changements dynastiques ${ }^{55}$. Un Père de l'Eglise comme saint Augustin a même considéré ces prophéties comme plus importantes que les prophéties bibliques. Au XII ${ }^{\mathrm{e}}$ siècle, le philosophe Abélard affirme explicitement que les Sibylles devaient leur autorité prophétique à leur virginité ${ }^{56}$. L'insertion de la prophétie sibylline Tiburtienne (Sibylla Tiburtina) dans le manuscrit de Fécamp représente donc un témoignage important de l'acceptation par la cour normande de l'autorité de Sibylle, soutenue par l'abbé Jean et indirectement par Guillaume de Volpiano.

En conclusion, les démarches historiographiques d'Adeliza de Bourgogne et des deux Adélaïde d'Aumale signalent que la préservation de l'information historique n'était pas exclusivement une tâche masculine. Le processus de collecte et de conservation des informations sur le passé découle d'une interaction entre les deux sexes. De la même manière, la connaissance et l'interprétation du futur résultent d'une collaboration entre les hommes et les femmes. Les femmes de l'aristo-

53. NORTH, 1987, p. 147-161.

54. GND, I, p. LXXXIV-LXXXV.

55. MC GINN, 1985, p. 7-35.

56. ABELARD, Epistola, éd. Jacques-Paul Migne, PL 178, c. 246-7. 
cratie, et parmi elles surtout les mères, avaient besoin de renseignements fiables sur le futur. Bien que le futur fût dans la main de Dieu, des abbés se faisaient les sincères interprètes de prophéties destinées à ces femmes. Guillaume de Volpiano, pour sa part, connaissait l'importance de la prophétie. Selon son biographe, Raoul Glaber, il dut son destin d'ecclésiastique à sa mère Perinza qui, à la suite d'un rêve prophétique, décida que Guillaume était destiné au service de Dieu ${ }^{57}$. Ce thème est un lieu commun de l'hagiographie, mais il souligne la croyance de la femme aristocratique aux prophéties aussi bien que le rôle des mères en tant qu'informatrices, et parfois de prophètes, de l'histoire de leurs enfants.

\section{Bibliographie}

Adso Dervensis de ortu et tempore antichristi necnon et tractatus qui ab eo dependunt, éd. Daniel VerHelst, Corpus Christianorum continuatio Mediaevalis, XLV, Turnhout, Brepols, 1996, 185 p.

BAtes, David, Regesta Regum Anglo-Normannorum. The Acta of William I (1066-1087), Oxford, Clarendon Press, 1998, XXXVIII + 1153 p.

BAUDUIN, Pierre, La frontière Normande aux $X^{e}-X I^{e}$ siècles, origine et maîtrise politique de la frontière sur les confins de la Haute-Normandie (911-1087), Caen, 1998, 562 p. (à paraître sous le titre: La Première Normandie ( $X^{e}-$ $X I^{e}$ siècles). Sur les frontières de la haute Normandie: identité et construction d'une principauté, Presses Universitaires de Caen)

BAUDUIN, Pierre, « Du bon usage de la dos dans la Normandie ducale (X $\mathrm{X}^{\mathrm{e}}$-début XII siècle) », communication présentée lors de la rencontre « Les transferts patrimoniaux durant le Haut Moyen Age (II) Morgengabe, dos tertia... et les autres organisée par l'Université de Lille III (CRHEN-O), l'Université de Valenciennes, l'Ecole Française de Rome et l'Università degli studi di Padua (Villeneuve-d'Ascq-Valenciennes, 2-4 mars 2000) [à paraître].

Bulst, Neithard, "Rodulfus Glabers Vita domni Willelmi abbatis ", in Deutsches Archiv für Erforschung des Mittelalters, 30, 1974, p. 450-487.

CORBET, Patrick, Les saints ottoniens : sainteté dynastique, sainteté royale et sainteté féminine autour de l'an mil, Beihefte der Francia, 15, Sigmaringen, Jan Thorbecke Verlag, 1986, 288 p.

DAviD, Charles W., Robert, Curthose, duke of Normandy, Cambridge Mass, Harvard University Press (Harvard Historical Studies, 25), 1920, XIII + 271 p.

Delisle, Léopold, Histoire du château et des sires de Saint-Sauveur-Le-Vicomte suivie de pièces justificatives, Valognes-Paris-Caen, 1867, 368 p.

Domesday Book seu Liber Censualis Wilhelmi primi regis Angliae, éd. A. FARLEY, Londres, 1783, 2 vol.

De moribus et actibus primorum Normanniae ducum auctore Dudone Sancti Quintini decano, éd. Jules LAIR, Caen, Mémoires de la Société des Antiquaires de Normandie, t. XXIII, 1865, 320 p.

57. Rodulfus, p. 258-259. 
Dugdale, William, Monasticon anglicanum, éd. John CALEY, Henry ElLIS et Bulkely BANDINEL, Londres, 6 vol., 1817-1820.

ENGLiSH, Barbara, The Lords of Holderness 1086-1260. A Study in Feudal Society, Oxford, Oxford University Press for the University of Hull, 1979, XII + 275 p.

FARRER, William, Early Yorkshire charters, III, Edinburgh, Ballantyne, Hanson and Co., 1916, XXXII + 501 p.

Fauroux, Marie, Recueil des actes des ducs de Normandie de 911 à 1066I, Caen, Mémoires de la Société des Antiquaires de Normandie, t. XXXVI, Société d'Impressions Caron et Co., 1961, 560 p.

The Gesta Guillelmi of William of Poitiers, éd. Ralph Henri Carless DAvis, Marjorie Chibnall, Oxford, Clarendon Press (Oxford Medieval Texts), 1998, XLVII $+199 \mathrm{p}$

The Gesta Normannorum Ducum of William of Jumièges, Orderic Vital and Robert of Torigni, éd. Elisabeth VAN HOUTS, Oxford, Clarendon Press (Oxford Medieval Texts), 1992-1995, 2 vol., CXXXIII + 156 p, XV + 341 p.

William of Malmesbury Gesta Regum Anglorum, éd. Roger Aubrey Baskerville MYNORS, Rodney ThOmson, Michael WinterbotTom, Oxford, Clarendon Press (Oxford Medieval Texts), 1998-1999, 2 vol., XXXII +879 p., XLVII $+496 \mathrm{p}$.

HunEYCUTT, Loïs, «The idea of the perfect princess : the Life of St Margaret in the reign of Mathilda II (1100-1118) », in Anglo-Norman studies, XII, Proceedings of the Battle conference, 1989, éd. Marjorie CHIBNALL, Woodbridge, Boydell Press, 1990, p. 81-98.

The Chronicle of John of Worcester, éd. Reginald Ralph DARLInGTON, Patrick MCGURCK, Jennifer BRAY, Oxford, Clarendon Press (Oxford Medieval Texts), 1995-1998, 2 vol., II et III, LXXXVI + 717 p., XIV + 351 p.

Johnson, Penelope D., Equal in monastic Profession. Religious Women in Medieval France, Women in Culture and Society, Chicago et Londres, The University of Chicago Press, 1991, XV $+294 \mathrm{p}$.

LE JAN, Régine, Famille et pouvoir dans le monde franc (VII ${ }^{e} X^{e}$ siècle). Essai d'anthropologie sociale, Paris, Publications de la Sorbonne, 1995, 571 p.

MCGINN, Bernard, "Teste David cum Sibylla: The significance of the Sibylline tradition in the Middle Ages", in Women of the Medieval World, Essays in honor of John H. Mundy, éd. Julius KIRSHNER, Suzanne F. WEMPLE, Oxford, Basil Blackwell, 1985, p. 7-35.

MUSSET, Lucien, « Recherches sur les communautés de clercs séculiers en Normandie au XI ${ }^{e}$ siècle », Bulletin de la Société des Antiquaires de Normandie, 55, 1959-1960, p. 5-38.

MusSET, Lucien « Recherches sur les pèlerins et les pèlerinages en Normandie jusqu'à la première croisade », Annales de Normandie, 12, 1962, p. 127-150.

Musset, Lucien, Les actes de Guillaume le Conquérant et de la reine Mathilde pour les abbayes caennaises, Caen, Mémoires de la Société des Antiquaires de Normandie, t. XXXVII, Société d'Impressions Caron et Co, 1967, 179 p.

NorTH, John D., "Some Norman horoscopes ", in Adelard of Bath. An English Scientist and Arabist of the Early Twelfth Century, éd. Charles BuRNETT, Londres, The Warburg Institute, University of Londres, 1987, p. 147-161. 
The Ecclesiastical History of Orderic Vitalis, éd. Marjorie CHIBNALL, Oxford, Clarendon Press (Oxford Medieval Texts), 6 vol., 1969-1978.

Platelle, Henri, «Les relations entre l'abbaye Saint-Amand de Rouen et l'abbaye Saint-Amand D'Elnone », in La Normandie Bénédictine au temps de Guillaume le Conquérant, préface par Gabriel URSIN LARGE, Lille, Facultés catholiques, 1967, p. 83-106.

The Chronicle of Robert of Torigni, éd. Richard HOwLETT, in Chronicles of the reigns of Stephen, Henry II, and Richard I, Rolls Series, Londres, Her Majesty's Stationary Office, 1889, 4 vol., IV, LXIX +419 p.

Rodulfus Glaber Opera, éd. France, John, BuLst, Neithard, ReYnolds, Paul, Oxford, Clarendon Press (Oxford Medieval Texts), 1989, CVI +323 p.

Round, John Horace, Calendar of Documents preserved in France illustrative of the history of Great Britain and Ireland, vol. 1, AD 918-1206, Rolls Series, Londres, Her Majesty's Stationary Office, 1899, LX + 680 p.

SHOPKOW, Leah, History and Community. Norman historical writing in the eleventh and twelfth centuries, Washington, The Catholic University of America Press, 1997, XII + 329 p.

Sibyllinische Texte und Forschungen, éd. Ernst SACKUR, Max Niermeyer HaLlE, 1898, $191 \mathrm{p}$.

STAFFORD, Pauline, «Cherchez la femme. Queens, queen's lands and nunneries : missing links in the foundation of Reading Abbey », in History. The Journal of the Historical Association, 85, 2000, p. 4-27.

STAPLETON, Thomas, " Observations on the history of Adeliza, sister of William the Conqueror », in Archaeologia, 26, 1836, p. 349-360.

VAN HOUTS, Elisabeth « Women and the writing of history in the early Middle Ages : the case of Abbess Matilda and Aethelweard », in Early Medieval Europe, 1, 1992, p. 53-68.

VAN HOUTS, Elisabeth, «The Brevis relatio de Guilelmo nobilissimo comite Normannorum written by a monk of Battle Abbey ", Chronology, Conquest and Conflict in Medieval England, Camden Miscellany, 34, Camden Fith Series, 10, Cambridge, Cambridge University Press for the Royal Historical Society, 1997, p. 1-48.

VAN HouTs, Elisabeth, Memory and Gender in Medieval Europe 900-1200, Basingstoke, Macmillan, 1999, XII + 196 p.

VAN HOUTS, Elisabeth, "L'oralité dans l'hagiographie normande au $\mathrm{XI}^{\mathrm{e}}$ et XII ${ }^{\mathrm{e}}$ siècles ", in Les Saints dans la Normandie médiévale, Actes du colloque de Cerisy-la-Salle (26-29 septembre 1996), éd. BoutT Pierre, Neveux Francois, Caen, Presses Universitaires de Caen, 2000, p. 83-94.

VAN HOUTS, Elisabeth, "L'écho de la conquête dans les sources latines : la duchesse Mathilde, ses filles et l'énigme de l'enfant doré ", in La Tapisserie de Bayeux: l'art de broder l'histoire, Actes du colloque de Cerisy-la-Salle (710 octobre 1999), éd. Brian LEVy, François NeveuX, Caen, Presses Universitaires de Caen, 2002, [à paraître].

Vita et Passio Waldevi comitis, éd. Rév. D. GILES, in Original lives of AngloSaxons and others who lived before the Conquest, Publication of the Caxton Society, XVI, Londres, 1854, p. 1-30. 
Walmsley, John, Charters and Custumals of the Abbey of Holy Trinity, Caen. Part 2 The French Estates, Records of Social and Economic History, new series, 22, Oxford, Oxford University Press for the British Academy, 1994, XII $+160 \mathrm{p}$.

WERTHEIMER, Laura, "Adeliza of Louvain and Anglo-Norman queenship », in The Haskins society journal. Studies in Medieval History, 7, 1995, p. 101-116. 


\section{Annexe}

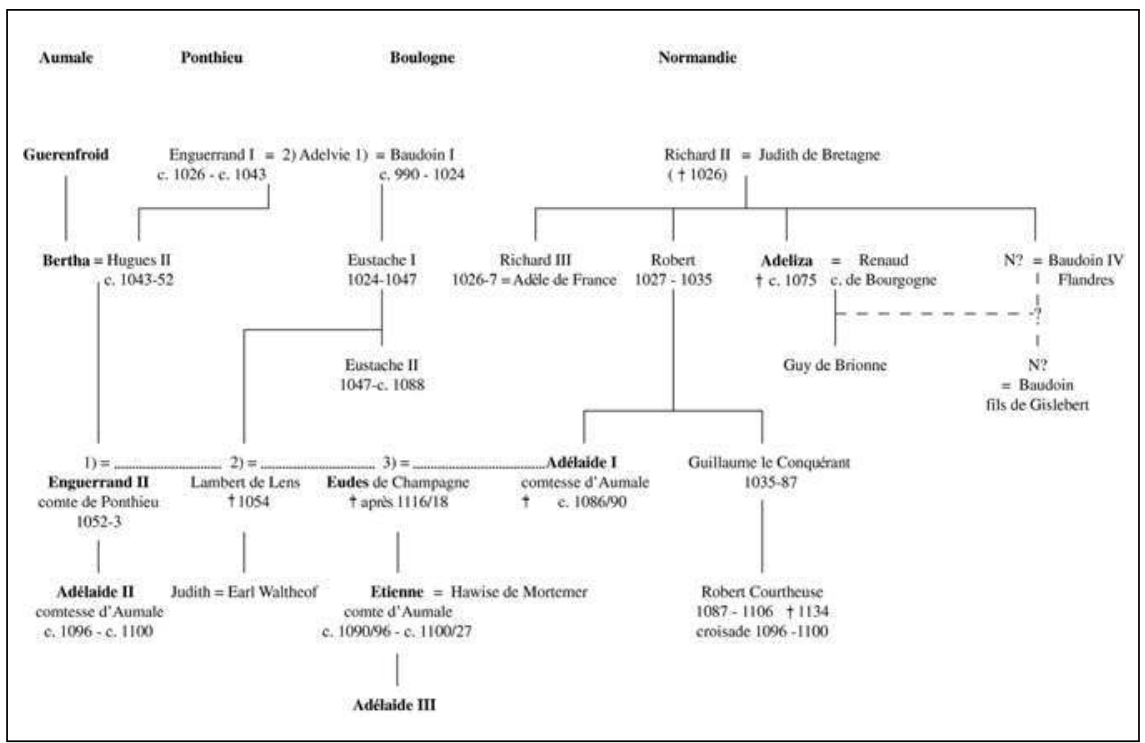

Les connexions familiales d'Adélaïde d'Aumale 